\title{
Proper motion of solar filaments
}

\author{
P. Ambrož ${ }^{1}$ and A. Schroll ${ }^{2}$ \\ 1 Astronomical Institute, Academy of Sciences of the Czech Republic, 25165 Ondřejov, Czech Republic \\ 2 Kanzelhöhe Solar Observatory of the University of Graz, 9521 Treffen, Austria \\ e-mail: schroll@solobskh.ac.at
}

Received 31 January 2001 / Accepted 8 August 2001

\begin{abstract}
Movement of solar filaments relative to the Carrington reference system is considered to be an appropriate quantity to characterize the horizontal transport of magnetic flux in the solar photosphere and chromosphere. A new method of measurement of both zonal and meridional velocities was developed using an extensive set of $\mathrm{H} \alpha$ observations. The velocity field was derived for many filaments during a few consecutive days and for different Carrington rotations. The proper motion of different parts of the filament relative to the Carrington reference system was determined. A velocity variation with a time-scale of about 10 hours was discovered. The presence of a non-axially symmetric horizontal velocity component in the filamentary motion was confirmed. The latitudeand longitude-dependent distribution of the horizontal velocity field was detected. The relationship between the horizontal velocities of solar filaments and the horizontal velocity field derived from background solar magnetic fields was found. In this paper the sources of errors are also analyzed in detail. Many measurements were disqualified by ill-defined contours of the filaments. The systematic errors, caused by the unknown height and shape of the filaments, are estimated. Only velocity values more accurate than $100 \mathrm{~m} \mathrm{~s}^{-1}$ were used in the study. The deviations of the mean horizontal velocities of filaments, magnetic field and sunspots resulting from the selection effect in their position on the Sun were interpreted.
\end{abstract}

Key words. Sun: filaments - magnetic fields - rotation

\section{Introduction}

Measurements of solar rotation show a difference (Van Tend \& Zvaan 1976) between the mean rotation rates of filaments, sunspots, the background magnetic flux and plasma in the solar photosphere. Filaments are usually considered to be good "tracers". Well-developed long-lived quiescent filaments usually cover a broad range of latitudes and longitudes for many solar rotations. There is a close relationship between the occurrence of filaments and the location of the inversion line between the opposite polarities of the background magnetic field in the photosphere. The filaments consist of plasma threads with chromospheric parameters, but the position of this phenomenon is dominantly determined by the distribution of the magnetic field in the photosphere. Not only the internal structure of quiescent filaments, but also their external shape may vary temporarily.

Send offprint requests to: P. Ambrož, e-mail: pambroz@asu.cas.cz

\section{Formulation of the problem}

The occurrence of the solar filaments on the neutral line between two magnetic regions with opposite polarity was first mentioned by Babcock \& Babcock (1955). Since then a set of different models of solar filaments has been constructed (Kippenhahn \& Schlüter 1957; Kuperus \& Raadu 1974; Lerche \& Low 1980). All agree that the occurrence of filaments is observed in the thin vertical slab above the neutral line between the pair of the background magnetic regions of opposite polarity. Displacement of filaments in the horizontal direction occurs either along the neutral line, or is closely related to its horizontal displacement as whole.

Due to the close relationship between the position of the filament and the location of the neutral line of the background magnetic field, the abovementioned difference between the rotation rates of filaments and of photospheric phenomena is not well justified by the different rotation rates of chromosphere and photosphere. We consider that the local displacement of the filaments can be due to the internal turbulent motion, changes in hydrogen ionization rate or change of the tilt in respect to the local vertical, 


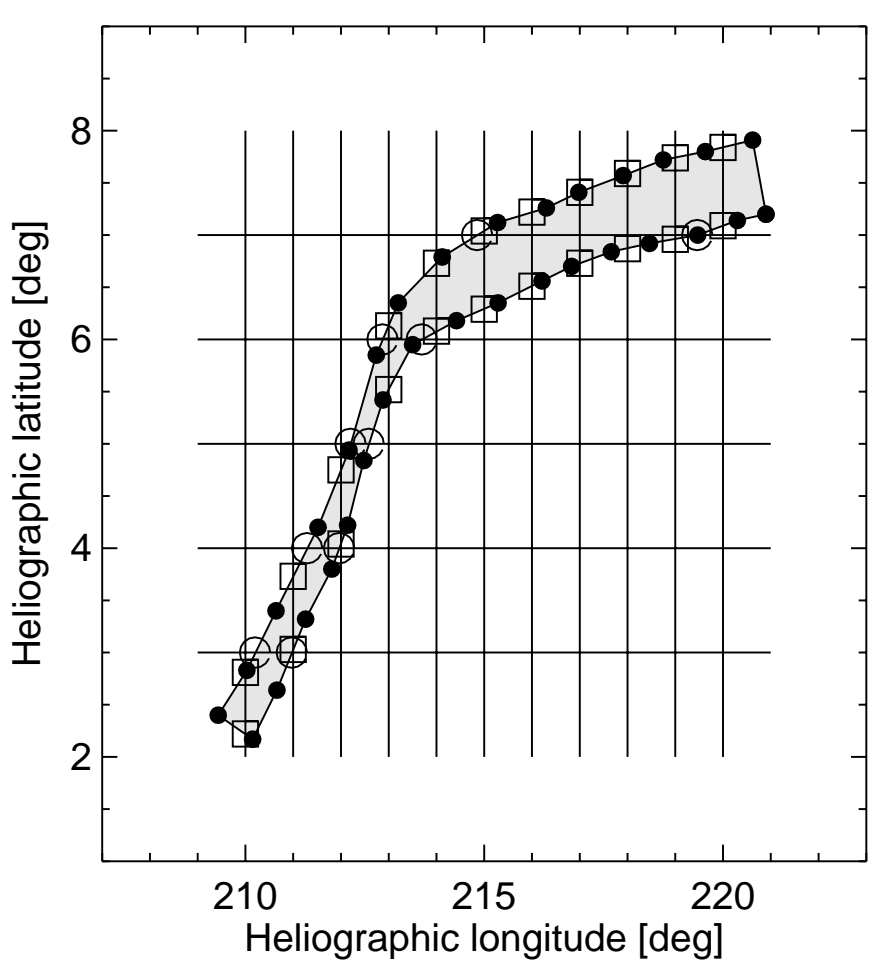

Fig. 1. Scheme of the measured contour points around one filament. Straight lines connect the individual points (the black bold circles). The new positions for integer values of the longitude (square boxes) and latitude (circles) were determined after co-ordinate transformation into the Carrington reference system.

resulting in apparent displacement. The cause of such discrepancies may also be in the systematically different horizontal zonal velocities at different positions in the solar photosphere. Local displacement is then related to the movement of the neutral line.

Using solar filaments to detect horizontal velocities seems to be effective mainly due to the large horizontal extent of these phenomena in heliographic longitude and in latitude up to the high polar regions. The filaments are characterized by a long lifetime; however, the long-lived reference points are absent from the filamentary body. The aim of our project was to determine the zonal and meridional velocities of filaments with maximal available accuracy. The purpose of the present study was to confirm whether it was necessary to consider in the individual cases only the axially symmetric (uniform in longitude) zonal flow of magnetized plasma at each latitude, or whether the displacement or movement of the filaments at different heliographic longitudes of one heliographic latitude was different. Finally, we would like to obtain the answer to the question of whether the proper motion of solar filaments and also of the general acceptability of filaments as 'tracers' in the study of horizontal flow in the solar photosphere and chromosphere. The project of measurements was focused on three time intervals; two during the phase of cycle maximum in the years 1980 and 1989 and one in 1986, in a phase of cycle minimum. Due to

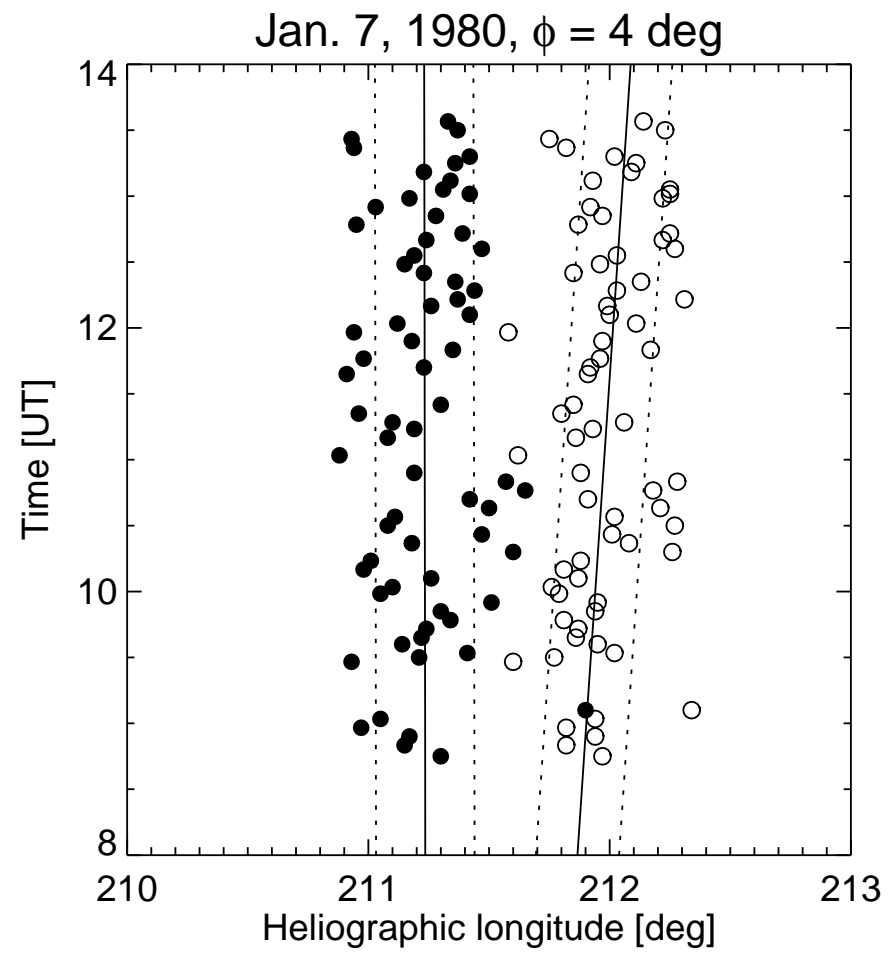

Fig. 2. Representation of the velocity determination due to the multiplied measurements of the left and right edge of the filament at latitude $4^{\circ}$ during the nearly 5-hour time interval, containing the measurements of the same filament on 71 different pictures. The straight lines, fitted using the least-squares method, form the basis for the determination of the zonal velocity and the corresponding error (the one $-\sigma$ limit is plotted as the dotted line), derived from the scatter of the individual measurements. The black circles are related to the values from the left contour of the filament.

the strict selection of only the precise results, explained in the next section, the final period of the measurements used covers the period of the solar cycle maximum from January to March 1980.

\section{Method of measurements}

The daily $\mathrm{H} \alpha$ patrol observations were made at the Kanzelhöhe Solar Observatory with an automatic chromospheric telescope having an entrance aperture of $100 \mathrm{~mm}$ and focal length of $2000 \mathrm{~mm}$. The telescope is equipped with a Halle $\mathrm{H} \alpha 0.07 \mathrm{~nm}$ pass band filter. Pictures of the solar chromosphere were taken every 4 min with an exposure time of about $1 / 100 \mathrm{~s}$. The mean original solar image on the film is about $18 \mathrm{~mm}$ in diameter. The image of the chromosphere with one or more well-developed filaments, selected for the measurements, was projected onto the measuring plane of the electronic digitizer. The mean diameter of the enlarged picture of the Sun was about $220 \mathrm{~mm}$. Manual measurements were made with the cursor in the laboratory reference system. The positions of at least 40 points measured around the solar limb and the trial reference points on the frame borders were first measured for each frame. The reference points were fixed 
with the mechanical telescope and camera systems and were used effectively to determine the position angle in calculating the heliographic co-ordinates.

The selection of filaments for further measurements depended, in the first instance, on the well-defined shape of the filament. Latitude and longitudinal displacements were considered, because it was possible to determine the displacement velocity for an extensive surface area. We used a series of pictures, taken during the whole observing day, in which dramatic changes in the shape of the filament were not observed.

The orientation of the solar image, the solar radius, as well as the central meridian position, was calculated. The data do not show any systematic changes of the measured position due to possible errors in the orientation of the instrument.

The selected filament was then measured at many points (usually around 100 measurements) around the contour. Usually more than one filament in each frame was measured in a similar way. This routine was repeated for the same filament many times during the whole observing day, depending on the length of the observing series (from 20 to 155 observations). Similar measurements were also made on consecutive days.

The position of subjectively selected points, however, does not coincide from frame to frame. This problem is due to the character of the observing material, where careful scrutiny disclosed no long-lived reference points in the filament. Therefore, the co-ordinates of the measured points were transformed into spherical heliographic coordinates. The heliographic co-ordinates on the solar disc were calculated using the standard procedure, developed earlier for Kanzelhöhe Observatory by one of the authors (A. S.). In the next step, the discrete and subjectively selected positions were homogenized.

All the points measured on each contour in the heliographic co-ordinate system were connected by straight lines. The intersection points either with parallels or with meridians, labeled by the integer values with an increment of $1^{\circ}$, were calculated and the comparable data sets in the heliographic co-ordinate system were obtained. The scheme of the measurements and the definition of the intersection points is shown in Fig. 1. Thus the procedure in which the measured points are connected by straight lines is justified only if the points are sufficiently close to one another, and if the filament contour between two measured points is sufficiently smooth. Both conditions were indeed satisfied during the primary measuring procedure. Sikora (1987) also tested the possibility of using cubic splines to connect the points, but the final effect did not lead to the improvement of the results; the numerical procedure only became more complicated.

The values of the corresponding individual heliographic longitudes or latitudes were plotted versus the time for each measuring day. The fit of all points by the regression line allow the mean horizontal (zonal or meridional) velocity and the corresponding error of the result for the whole day to be determined. This step in the
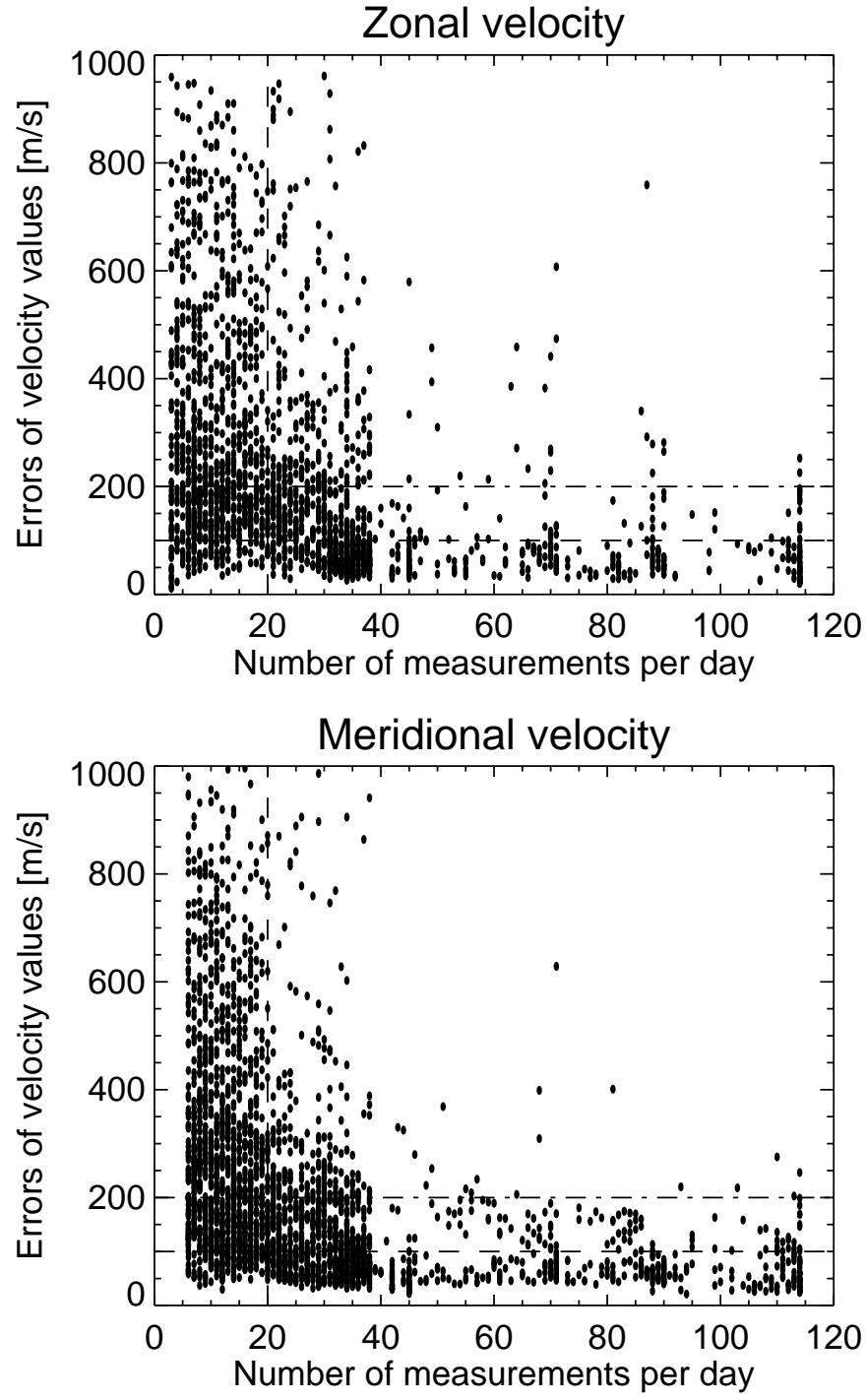

Fig. 3. Test of the relationship of the dependence of the velocity errors on the number of measurements for zonal and meridional velocities. Of all the measurements only the velocity values determined from 20 or more measurements with the errors lower than $100 \mathrm{~m} \mathrm{~s}^{-1}$ were considered. See discussion in the text.

determination of the daily averaged velocity and of the standard deviation limits is illustrated in Fig. 2.

\section{Results and discussion of the velocity measurements}

The calculation of the daily averaged velocity values makes it possible also to assign each velocity value an error estimate, derived from the standard deviation of all fitted points. We are able automatically to obtain the relationship between the number of the measured points and the errors of the velocity values. The scatter plot of all estimated errors is presented in Fig. 3 for both the zonal and meridional velocities. Clearly, the low number of measurements per day (under 20 measurements) yields the results with a dominantly large scatter of errors. According 


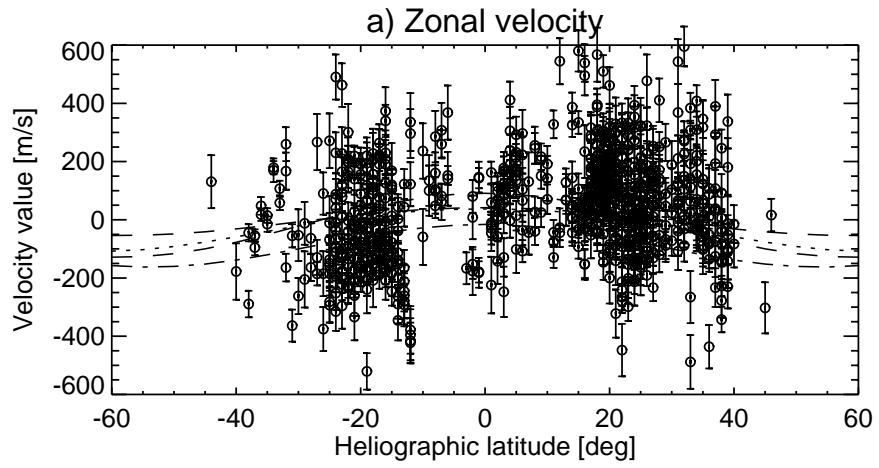

b) Mean zonal velocity

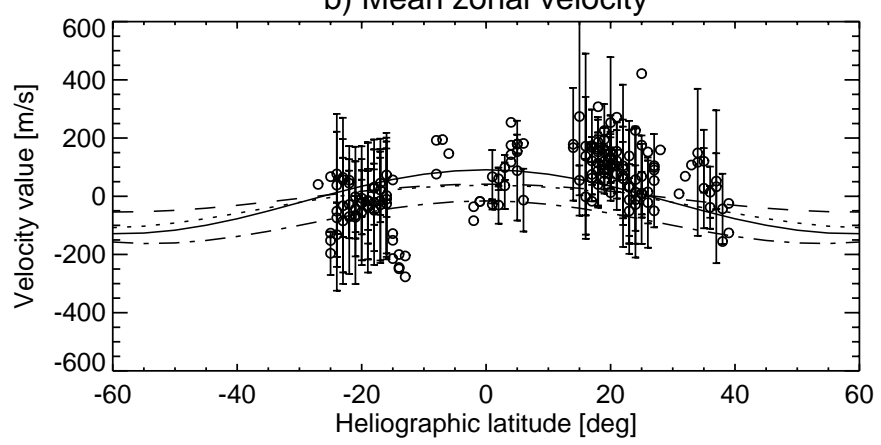

c) Meridional velocity

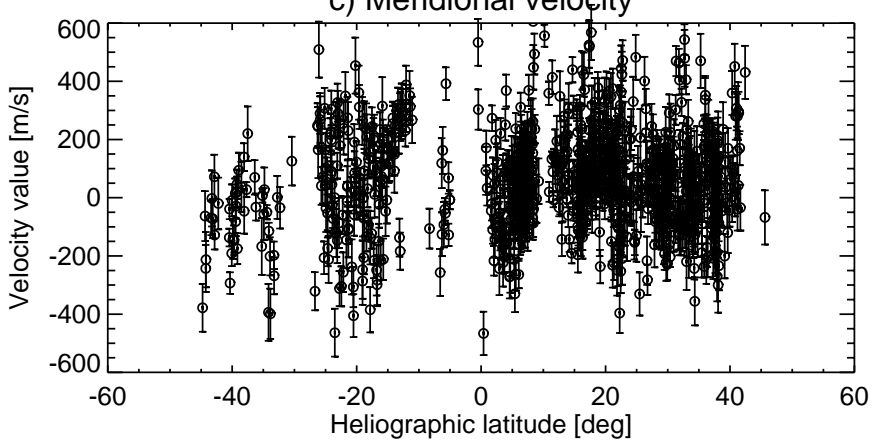

Fig. 4. Latitude distribution of measured zonal a) and meridional c) velocities. The scatter of the individual velocity values from the axially symmetric rotation rate curves, derived by Glackin (1974) - dotted, Adams \& Tang (1977) - dashed, Brajša et al. (1991) - solid, and by Japaridze \& Gigolashvili (1992) - dash dot, demonstrate the presence of the non-axially symmetric component of the velocity field, oriented zonally or meridionally. The errors are computed from the all days measurements. Latitude distribution of the mean zonal velocities b) is averaged over two or more days, respectively. The error bars represent the errors of the arithmetic means and demonstrate the scatter of the velocity values in the averaging interval.

to our tests, the least-squares value with very small errors, obtained in a few measurements, cannot be accepted for serious argumentation either, because the alignment of the measuring points can be random. Therefore, we used velocity values, computed from at 20 measurements per day. The upper limit of the errors was taken at $100 \mathrm{~m} \mathrm{~s}^{-1}$. This data "filter" eliminates a large number of measurements, but our velocities have a high standard of accuracy.
The one-day observing period does not exceed 6 hours, but is usually shorter. The total number of measured daily mean velocities is 1051 . Later the number of daily mean velocity values was reduced to 546 , after only the measurements with errors not exceeding $100 \mathrm{~m} \mathrm{~s}^{-1}$ were selected for our study.

The latitude dependence of the zonal and meridional velocities relative to the Carrington reference system is presented in Fig. 4. Usually this kind of presentation is used to determinate the zonal averaged zonal or meridional velocities. The scatter plot of the zonal velocity values was combined with the set of fitted curves of the rotation laws, derived by different authors (Glackin 1974; Adams \& Tang 1977; Brajša et al. 1991; Japaridze \& Gigolashvili 1992). The scatter of the curves is lower than $200 \mathrm{~m} \mathrm{~s}^{-1}$, but the scatter of the individual velocity values is nearly $800 \mathrm{~m} \mathrm{~s}^{-1}$, although the internal accuracy of the measured points at the filament's edge is only $100 \mathrm{~m} \mathrm{~s}^{-1}$. A similar situation also applies to the meridional velocities. From these differences one can conclude that the possible axially symmetric flow is combined with a much more spatially variable velocity field, dependent on the latitude and longitude position on the solar surface.

The zonal average of the zonal velocity we usually interpreted in terms of the symmetric component of the zonal velocity or the rotation rate. Measurements of the individual filament displacement produce velocities averaged over one observing day. The filaments, for which velocity measurements during an interval of between two and four consecutive days were available, were selected, and the average values were computed again and presented in Fig. 4b. The error bars in this case represent the error of the arithmetic mean and relate with the scatter of the daily velocity values on these few averaged days. The scatter is in many cases greater than internal accuracy of the daily measurements. The axially symmetric rotation rate curves of other authors are the same as in Fig. 4a. The distribution of velocities also demonstrates the deviation of the local velocities from the rotation rate. The comparison of Figs. $4 \mathrm{a}$ and $\mathrm{b}$ shows that the daily values and the values averaged over a few days deviate from the rotation rate. The daily local velocities are superposed on the average local velocities and both are superimposed on the mean rotation velocity. The velocities presented in Fig. 4 show the possible N/S asymmetry, mainly for zonal displacement. This effect is true for the presented set of data. However, we consider that in our case it is only as occasional selection effect, due to the limited amount of filaments, measured during the relatively short time interval. It is very difficult to make serious general conclusions about N/S asymmetry from our data. The diversity of the rotation rate values according to different authors is very probably caused mainly by the sampling effect or by different methods of measurement used.

The results of the measurements of the individual filaments made during the few days are presented in Fig. 5, where the velocity values and the errors for one filament are summarised. The corresponding velocities on the 

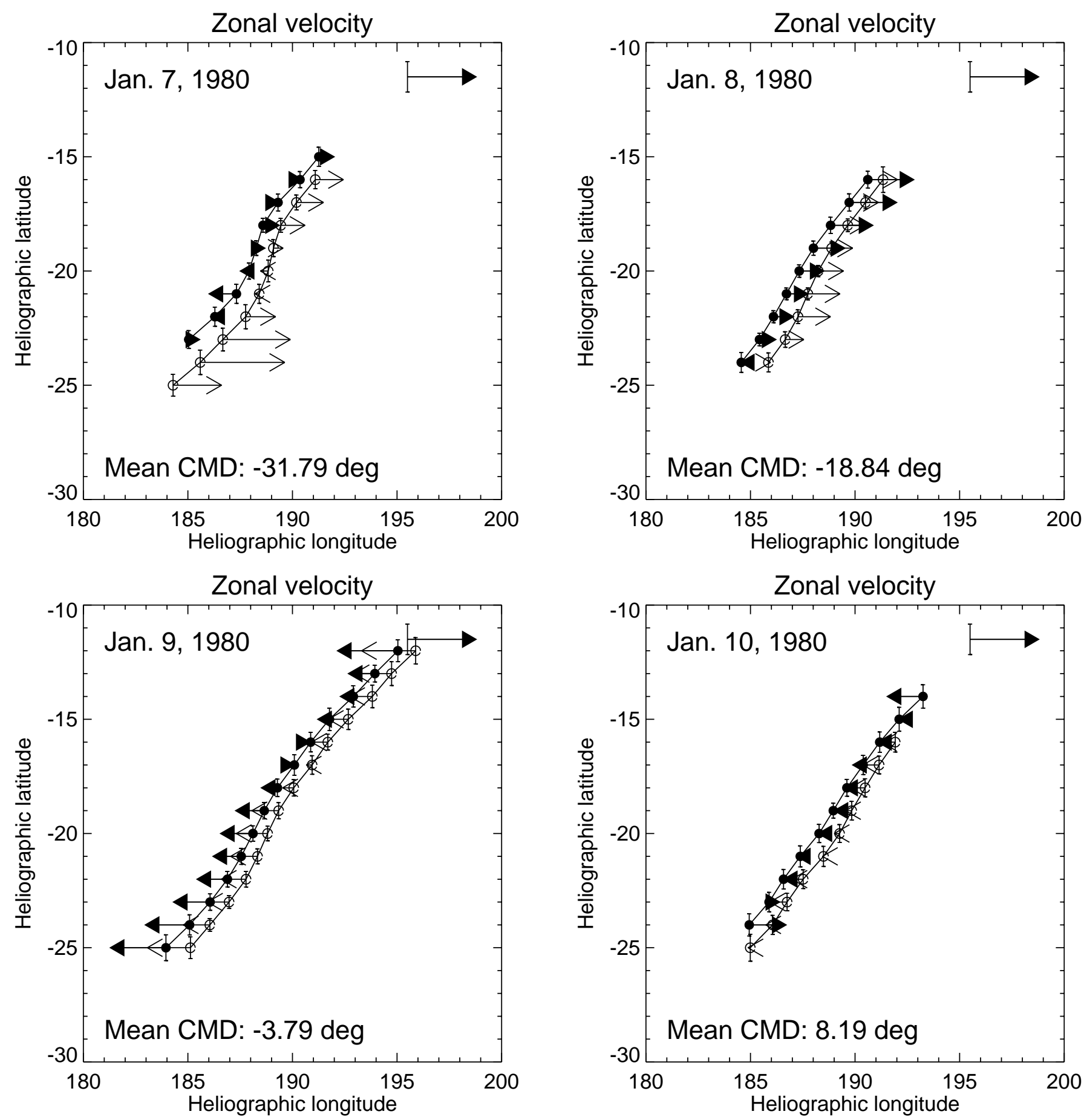

Fig. 5. Example of the zonal velocity field for one filament, measured over four days. The arrow in the upper right corner corresponds to the velocity of $500 \mathrm{~m} \mathrm{~s}^{-1}$ and the error bars to the error of $100 \mathrm{~m} \mathrm{~s}^{-1}$. Bold and thin arrows show values from the left and right contours of the filament, respectively. The filament points position and the mean Central Meridian Distance (CMD) are calculated for the mean time, derived from all observations, made during the day.

latitude circle and on the given side of the filament are time-dependent. The obtained velocities are also of different magnitude on the opposite sides of the filament and, in some cases, they also change their orientation. The velocity values are usually larger than the estimated errors and in many cases they exceed the value $100 \mathrm{~m} \mathrm{~s}^{-1}$. As documented in Fig. 6, similar properties are also typical for the meridional velocity fields. The different parts of the filament move with different velocities. Filaments shake (oscillate) perpendicularly to the filament axis, or make wave-like motions in the direction of the filament axis on the time scale of one day. The average velocity of all points also characterises the general displacement of the whole filament body. 

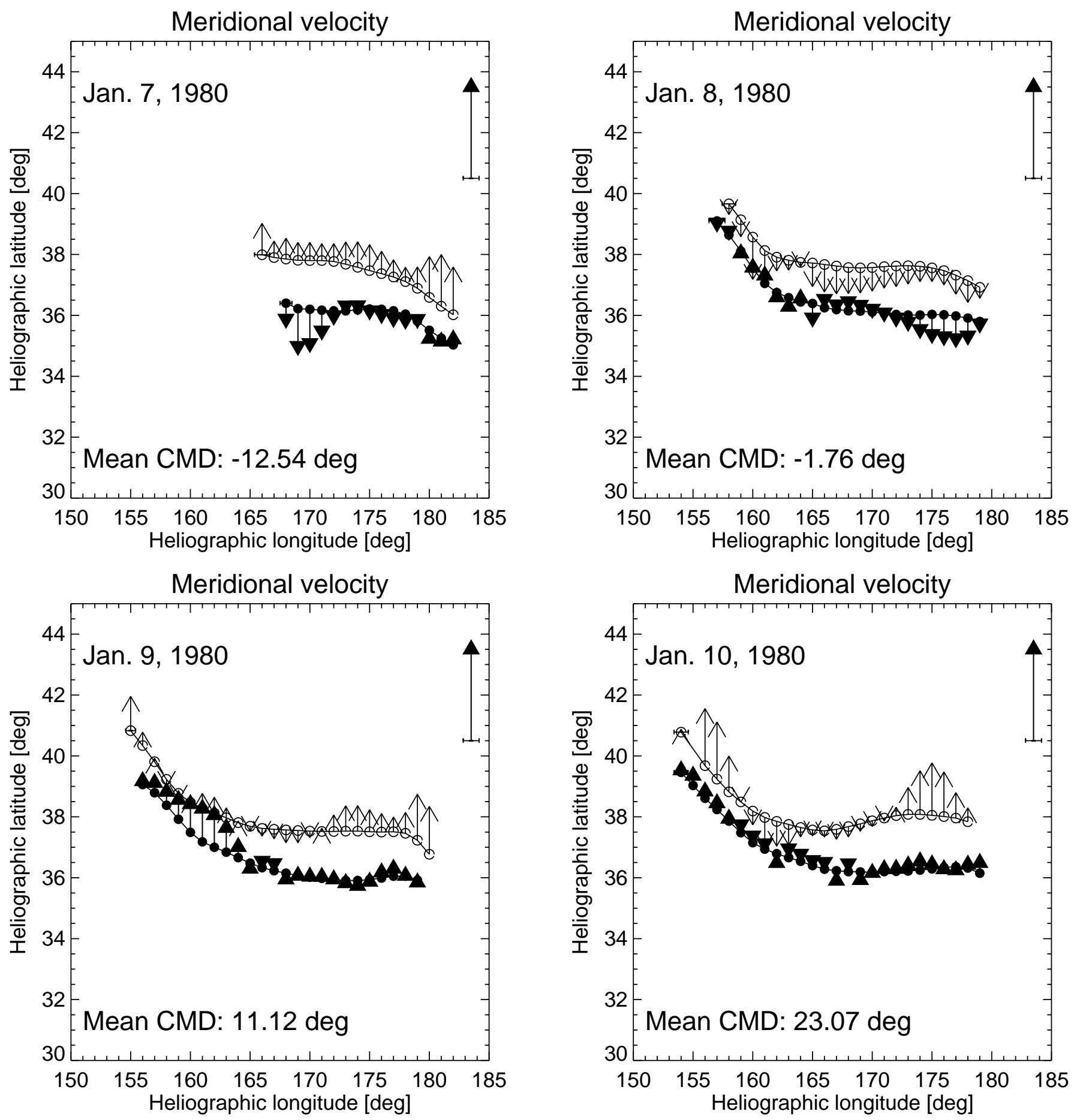

Fig. 6. Example of the meridional velocity field, measured over four days. The arrow in the upper right corner corresponds to the velocity of $500 \mathrm{~m} \mathrm{~s}^{-1}$ and the error bars relate to the error of $100 \mathrm{~m} \mathrm{~s}^{-1}$. Bold and thin arrows draw values from southern and northern contours of filament, respectively. The filament points position and the mean CMD are calculated for the mean time, derived from all observations, made during the day.

Filaments exist for a long time, many Carrington Rotation Periods (CRP), and, therefore, systematic displacements in different directions are detected. Usually, the fluctuation component also is superimposed over a short period. According to our measurements, the rotation of some filaments around "pivot points" confirms the results of Mouradian et al. (1986). They interpreted such points as a singularity of differential rotation. According to our results these points represent the centre of the vortex of large-scale horizontal flow of the magnetized plasma. 
The filament, being strongly determined by the magnetic structure in the solar photosphere, then rotates together with the magnetic flux distribution (neutral line) around such a point. The lifetime of the large-scale velocity structures is a few solar rotation periods and corresponds well with the time interval necessary for deriving the "pivot points".

\subsection{Influence of the vertical structure of the filaments}

The effect of the vertical structure of the filament on determining the rotation rate was studied by Roša et al. (1998). The measurements, carried out in the image plane, generally reduce the angular velocity and the angular displacement if the object is located at the height $h$ above the solar photosphere. This effect can be illustrated using the model expression, derived by Roša et al. (1998). The apparent zonal velocity $v_{\text {app }}$ due to this projection is

$v_{\text {zon }}(\lambda, \phi, h)=\frac{\cos \lambda}{\beta^{2}-\sin ^{2} \lambda} v_{\text {app }}$,

where $\lambda$ is the projected central meridian distance and $v_{\text {zon }}(\lambda, \phi, h)$ the resulting zonal velocity for the given coordinates of the measured points. If the rotation axis is perpendicular to the line of sight and for the simple point, the parameter $\beta$ is

$\beta=\frac{\left(1+\frac{h}{R_{\odot}}\right)-\sin ^{2} \phi}{\cos \phi}$,

where $R_{\odot}$ is the solar radius and $\phi$ the heliographic latitude projected onto the image plane. The results of this test, with apparent latitude $\phi=45^{\circ}$, are shown in Fig. 7. We postulate the apparent velocity $v_{\text {app }}=100 \mathrm{~ms}^{-1}$ and the zonal velocity $v_{\text {zon }}$ was calculated for heights $h=0,10,20, \ldots, 100 \mathrm{Mm}$. The resulting velocity decreases with increasing height $h$ and also with increasing apparent central meridian distance $\lambda$. One can see that the decrease of the zonal velocity in the range of $\lambda$ and $\phi$ not exceeding $45^{\circ}$ is no more than $30 \%$ of the apparent velocity if $h=100 \mathrm{Mm}$. According to the evaluation of our measured filaments, the usual height of our filaments is around of $30 \mathrm{Mm}$ and in such a case, the maximal decrease is about $10 \%$.

Application of the abovementioned test is problematic for two substantial reasons. The first problem is connected with the real height of the filament. On the solar disc we cannot determine the height directly and we usually approximate this value from the measurements at the east and west limb. It is, however, well known from cinematographic limb observations that quiescent prominences are also able to change their height substantially in the course of one day, and that these changes may be irregularly distributed along the filament body. The second problem is closely connected with the real shape of the filament in the sense of the profile perpendicular to the length axis of the filament. The above-mentioned test is derived for a point in coronal space and can be accepted also for a vertically oriented thin slab.
Departure from our results can be expected if the filament profile is of another shape, such as a circle, rectangle, triangle, arc or another more complex profile. The problem can be also complicated by the fact that the created spatial body of the filament does not lie simply in the chromosphere, but hangs on the magnetic field configuration in coronal space and is not visibly connected with the chromosphere, except at the feet. Such examples are probably much closer to reality than the simple thin slab, generally accepted in the theoretical approximation of filaments. The abovementioned complications justify the approximate evaluation of the possible error rather than including the correction algorithms directly in the procedure of computing the filament velocity. We consider the possible errors, caused by the vertical projection of filaments as the minority effect, when the horizontal displacement measurements are made not very far from the solar disc centre. The sophisticated determination of the height of the filament, as recommended in Roša et al. (1998) and Vršnak et al. (1999) can be used statistically correctly in the case of extensive measurements of filaments over a long time interval, where average values are sufficient. For our measurements and calculations on the short time scale of a few days, the height of the filament at the special point must be considered as a free parameter of the second order, having a similar influence on the daily velocity results as the real width or shape of the filament.

\section{Horizontal velocity derived from magnetic flux displacement}

The derivation of the horizontal transport velocity is made separately for the meridional and zonal direction. In the present paper only the zonal velocity was used. The velocity determination from the proper motion of filaments and from the displacement of large-scale magnetic field regions relates very probably with the similar substance, however, the velocity determination is made by different methods.

The zonal velocity field in the photosphere can also be derived from the temporal evolution of the largescale magnetic field distribution. Our data, measured with an instrument characterised by high sensitivity, but low spatial resolution, at the Wilcox Solar Observatory (WSO) of Stanford University, were additionally filtered by least-squares transformation into a series of spherical harmonic functions, truncated at the maximal principal index $l=12$. The small-scale features with a short lifetime are effectively filtered and only long-lived structures were used. The velocity structure, responsible for the time evolution of the large-scale magnetic flux, was inferred (Ambrož 1993, 2001a, 2001b) with the help of the "Local Correlation Tracking" method (November 1986) applied to a pair of consecutive magnetic synoptic charts. The derived velocity field is determined at each nodal point of the heliographic mesh with a point distance of $5^{\circ}$ in both meridional and zonal directions. The velocity map covers the whole photosphere and the inferred velocity field is 


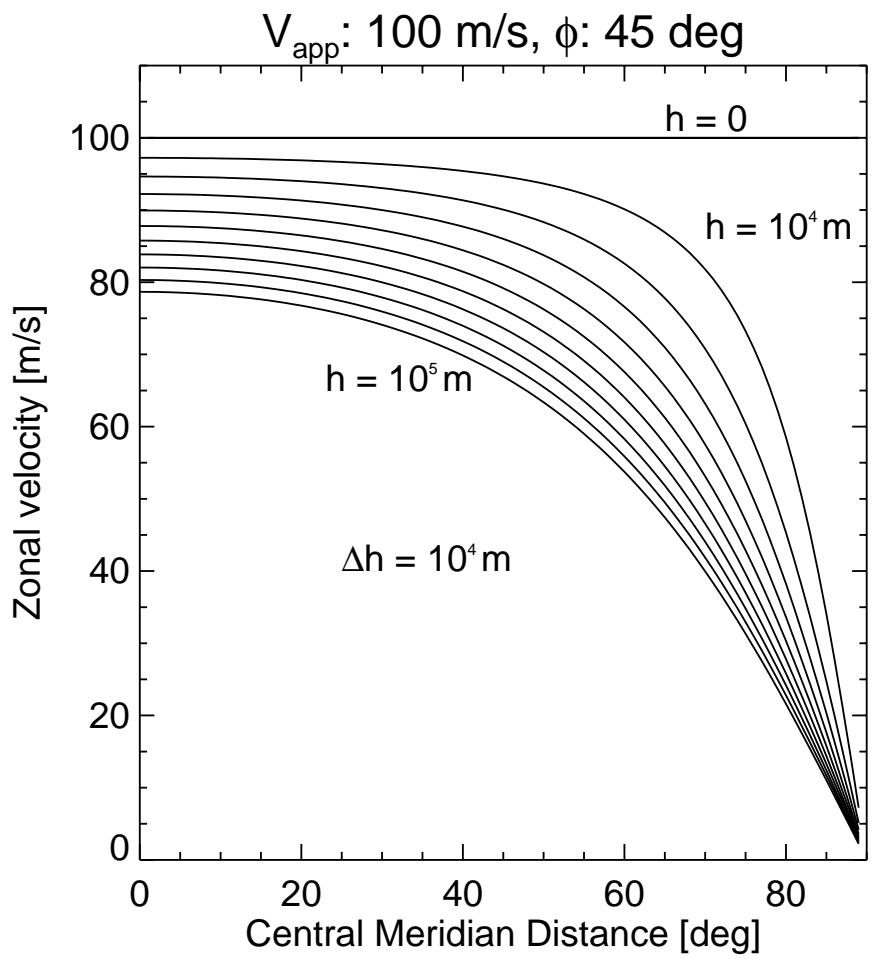

Fig. 7. Influence of the height structure of the stable thin slab filament on the measured zonal velocity. The constant line at $100 \mathrm{~m} \mathrm{~s}^{-1}$ is obtained for the measurements in the chromosphere for zero vertical velocity. The other curves are computed for increasing height above the chromosphere at increments of $10^{4} \mathrm{~m}$ up to the height of $10^{5} \mathrm{~m}$. The largest systematic error reduces the measured velocity for $27 \mathrm{~m} \mathrm{~s}^{-1}$ on the limited CMD $40^{\circ}$ and latitude $\phi=45^{\circ}$.

responsible for continuous displacements of the magnetic flux over several solar rotations. The large-scale flows are considered on the characteristic scale length from 20 to 60 heliographic degrees. This velocity field, however, does not contain information about the flow on a characteristic length scale, comparable to or lower than the dimension of supergranules. If one would like to compare the velocity results from magnetic field displacement and from the filaments' proper motion, it would be necessary to calculate the averages of the filament velocity values over all the points of each filament and over the time interval of many (or all) available days.

We used the magnetic flux displacement during Carrington rotation No. 1690. Two separate charts are shown: with the zonal velocity in Fig. 8 and with the meridional velocity in Fig. 9. In both cases the positions of the contour points of the filaments are plotted in the velocity chart.

The filament is a spatial phenomenon, projected into the image plane. The angular resolution is limited by the resolution of the telescope and by the seeing; the temporal resolution is of the order of seconds. The large-scale magnetic field is derived from synoptic charts. The synoptic chart is a composition of sectors selected from daily

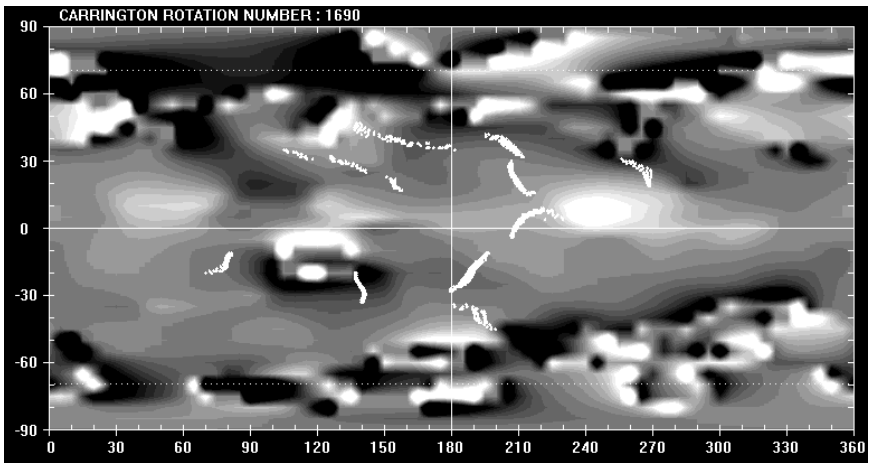

Fig. 8. Composition of the synoptic chart of the inferred zonal velocity distribution and the filament positions, measured during the CR 1690. The dark regions relate with the zonal velocity oriented in the sense of solar rotation, in the white regions the velocity vectors are oriented oppositely. The filament positions do not usually coincide with the location of the maximal zonal velocity.

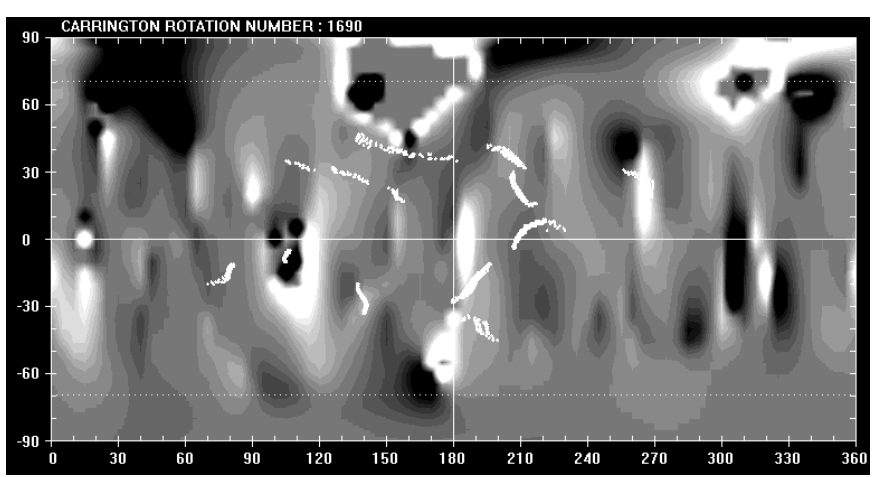

Fig. 9. Composition of the synoptic chart of the inferred meridional velocity distribution and the filament positions, measured during CR 1690. The dark regions relate with the meridional velocity oriented to the solar north pole; in the white regions the velocity vectors are oriented oppositely. The filament positions do not usually coincide with the location of the maximal meridional velocity.

observations near the central meridian and mounted in consecutive order during one Carrington rotation period. To obtain information during the whole CRP about the large-scale displacement, the small-scale phenomena with a short lifetime were omitted.

The inferred large-scale zonal velocities also have a non-axially symmetric component, and the velocity value varies over the solar photosphere. The filaments do not coincide with the regions of velocity extremes and are located in regions with a high zonal velocity gradient. The presence of the velocity gradient across the filaments is the probable cause of the deformation, if any, of the magnetic structure, supporting the filament. The occurrence of solar filaments seems to be a selective effect in relationship with the magnetic neutral line and with the special 
a)Mean values for individual filaments

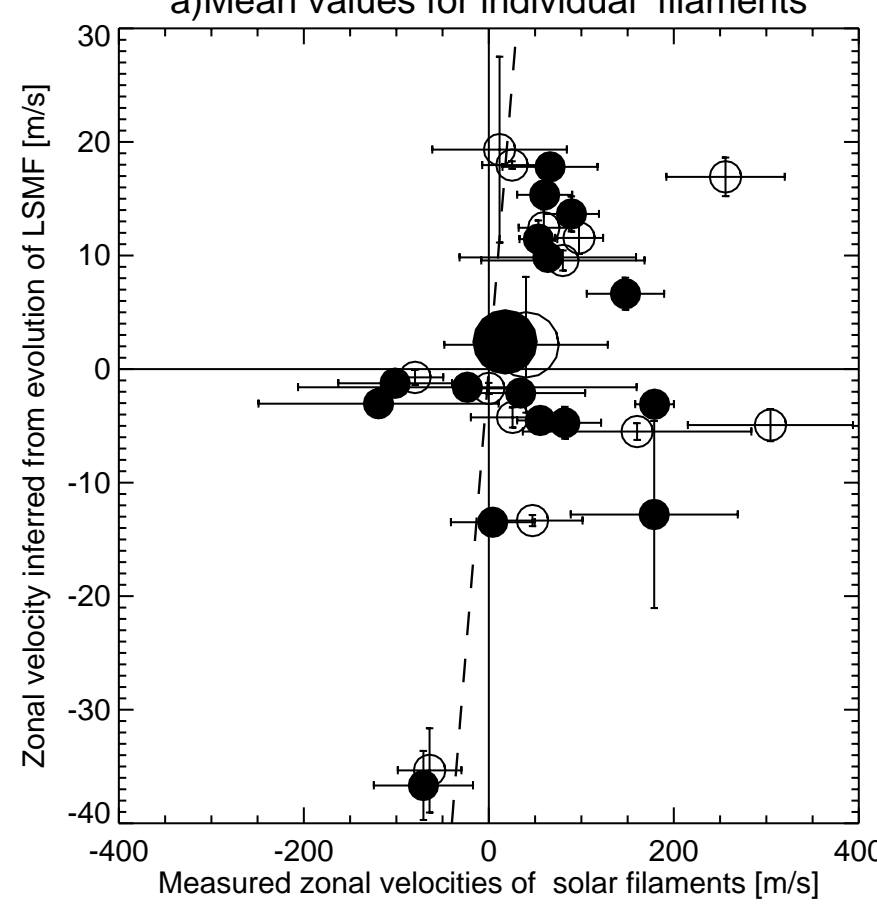

b)All filaments

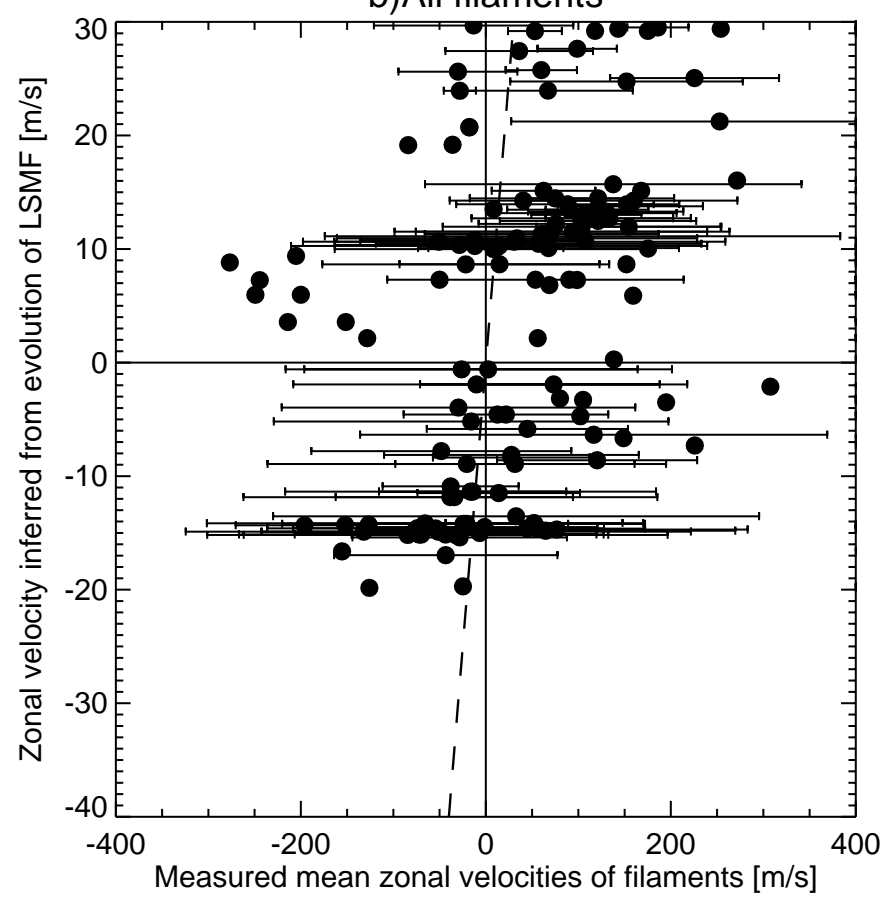

Fig. 10. a) The scatter plot of the corresponding mean values of zonal velocities, as derived from filament displacements and from the evolution of the large-scale magnetic field. The black and white circles relate with the left and right contours of the filament, respectively. The pair of large circles shows the positions of the total averages of all measurements. The mean values for both the velocity sets are computed for each measured filament and to the corresponding velocity value, inferred from the magnetic flux displacement. b) The scatter plot of the relationship between the available mean values of the zonal displacement of filaments and the corresponding zonal velocities derived from the magnetic flux displacement. The error bars represent the error of the arithmetic mean and relate with the scatter of the velocity values during the averaging time interval. velocity structure, which is non-axially symmetric over the whole solar sphere.

Two arrays of corresponding velocities are plotted in Fig. 10. The scatter of the individual velocity values is much greater for each filament than the scatter of the corresponding values, inferred from the magnetic field evolution. Both velocity fields are different in nature. The filament measurements produce the temporally dependent velocity field. The cause is the short time scale of the filament measurements, in which the small-scale velocity fluctuations with short lifetime are not sufficiently filtered. A substantial number of the plotted points is located in the first and third quadrants. This supports our assumption of the large-scale magnetic field displacement due to the large-scale velocity flow. The proper motions of filaments relate proportionally with the displacement of the magnetic inversion line.

Another comparison of the measured zonal velocities of the filaments with the corresponding zonal velocity of the magnetic flux displacement is presented in Fig. 10b. Both sets of velocities relate to the Carrington reference system. We selected only the zonal velocity measurements of the filaments, measured during two or more consecutive days for a selected latitude. We have tried to eliminate the short-term changes of the velocity values caused by the small-scale velocity fields by averaging the values from the individual consecutive days. The error of the arithmetic mean of the filament velocity values relate with the scatter of the individual measurements in each data series. The dashed line represents the regression line for perfect correlation. The correlation coefficient of both velocity vectors is $r=0.272$. The indicated difference between the absolute velocity values of both data sets can be explained by different methods of measurement and by different averaging in the two cases. The magnetic flux displacement is measured from the displacement during one CRP, and the flux distribution is spatially smoothed by a spherical harmonic function with the maximal principal index $l=12$. The filaments were measured on daily patrol pictures and averaged over at most 4 days. The filament data contain much more from the local velocity component than the magnetic flux displacement data, in which the large-scale velocities dominate.

\section{Conclusions}

The following results were derived:

- The low accuracy of the individual measurements of filaments follows from their nature (diffuse objects without persistent reference points). According to our estimation, the error of the individually measured value of the velocity is probably higher than $1000 \mathrm{~ms}^{-1}$. Measurements from the many time intervals are difficult to compare, also due to the poorly defined measured points. Such potential inaccuracy must be generally expected in all measurements of solar filaments, 
made by a similar method from photographic pictures of the solar chromosphere.

- The average horizontal velocity of solar filaments can be measured with an accuracy better than $100 \mathrm{~ms}^{-1}$ relative to the Carrington reference system if many measurements during the day are available. Eliminated are also the velocity values, derived from individual measurements less than 20 in number if the calculated error is lower than our limit.

- The filament changes its shape irregularly from day to day, probably due to the underlying inhomogeneous velocity field, oriented in both the zonal and meridional directions. This character of the velocity field causes the shaking or waving of the filament body as a whole, or in parts.

- The local instantaneous velocities are usually much higher than the mean velocity of the whole filament, or the average velocity of the filament in the course of few days. The local velocities can be connected with the small-scale displacement of the magnetic flux ropes, supporting different parts of the filament.

- The individual velocity values plotted versus latitude display a much larger scatter than the mean latitude dependence of the rotation rate, derived by other authors. The rotation rate curves, determined by different authors, are also scattered, but the range of the scatter is lower than the scatter of the daily means. The errors of the daily mean values are significantly lower than the departure of velocity values from the average of the rotation rate curves. We consider this velocity to be the proper motion velocity of the solar filaments related to the Carrington reference system.

- Filaments are vertically oriented phenomena and their projection into the image plane can be a source of systematic errors, leading to the overestimation of the measured velocities. This effect can be critical near the solar limb. Our measurements, carried out closer to the disc centre than $40^{\circ}$, must be reduced by about $25 \%$ if the height of the filament is $100 \mathrm{Mm}$. These parameters are extremes, the values usually used are much lower (1/3 of the height) and, therefore, the errors are also smaller. In our results the temporal variability of the height and tilt of filaments is not considered.

- The zonal and meridional velocity fields, inferred from the horizontal displacement of the magnetic flux, have many of the local maxima. The positions of the filaments, however, never coincide fully with the positions of the inferred maxima. Filaments are distributed in regions with a relative maximum of the horizontal velocity gradient. Filaments are surrounded on both sides by flows, leading to shearing deformation.

- The comparison of the mean zonal velocities of the measured filaments, averaged over the whole time interval of measurements, and the zonal velocities, inferred from the magnetic flux displacement at the identical points, are aligned in our correlation graph from the first to the third quadrants. Thus distribution indicates the positive correlation of both velocity fields and supports the hypothesis of the displacement of filaments due to the displacement of the underlying magnetic field structure.

- The two previous conclusions indicate that the difference between the variety of the rotation laws on the Sun could be explained by the selection effect. Filaments are distributed only in specific regions on the Sun, where the horizontal velocity generally and the zonal velocity specially do not reach maximal values. The zonal mean from such regions is necessary apart from the zonal mean from regions, where the maximal regions are included. Statistically two systematically different data sets are investigated. The presence of the selection effect can be applied to many other "tracers", being closely connected with the magnetic field and special structures of the horizontal velocity field.

- Solar filaments can, in principle, be used as "tracers" for measurements of the velocity field in the solar photosphere and chromosphere, however, the task is limited by the extremely laborious procedure of the measurements. We are very skeptical of measurements based on a lower number of independent samples than 20 per day. Also, the simplifying of the filament localization by introducing a "center of filament" prior to the measurements at many points on the filament contour or in the filament body causes loss of filament identity and the results are confusing. The application of the strong condition of data selection according to accuracy leads to the elimination of many measured values. In our case of the initial 170 thousand measurements used, in Fig. 4b or in Fig. 10b, only 160 points we presented.

Acknowledgements. One of the authors (P. A.) is grateful to Prof. H. Haupt and Dr. A. Hanslmeier for their kind invitation and to the staff of Kanzelhöhe Solar Observatory for their hospitality during his stay, granted by the Austrian Academy of Sciences under agreement with the Academy of Sciences of the Czech Republic. The Wilcox Solar Observatory data used in this study were obtained via the web site http://quake. stanford.edu/ wso courtesy of J. T. Hoeksema. The authors are also grateful to X. Zhao for his kind help during the period of data preparation. The Wilcox Solar Observatory is supported by NASA, NSF, and ONR. The present study was made with the support of Grants Nos. A3003806 and S1003006 of the GA/AVČR and No. 205/01/0657 of the GAČR.

\section{References}

Adams, W. M., \& Tang, F. 1977, Sol. Phys., 55, 499

Ambrož, P. 1993, in The Magnetic and Velocity Fields of Solar Active Regions, ed. H. Zirin, G. Ai, \& H. Wang, Proc. IAU Colloq. 141, Beijing 1992, PASP Conf. Ser., 46, 495

Ambrož, P. 2001a, Sol. Phys., 198, 253

Ambrož, P. 2001b, Sol. Phys., 199, 251

Babcock, H. V., \& Babcock, H. D. 1955, ApJ, 121, 349 
Brajša, R., Vršnak, B., Ruždjak, V., et al. 1991, Sol. Phys., 133, 195

Glackin, D. L. 1974, Sol. Phys., 36, 51

Japaridze, D. R., \& Gigolashvili, M. Sh. 1992, Sol. Phys., 141, 267

Kippenhahn, R., \& Schlüter, A. 1957, Zs. f. Ap, 43, 36

Kuperus, M., \& Raadu, M. A. 1974, A\&A, 31, 189

Lerche, I., \& Low, B. C. 1980, Sol. Phys., 66, 285

Mouradian, Z., Martres, J.-M., Soru-Escaut, I., \& Gesztelyi, L. 1987, A\&A, 183, 129

November, L. J. 1986, Appl. Opt., 25, 392
Roša, D., Vršnak, B., Božič, H., et al. 1998, Sol. Phys., 179, 237

Sikora, G 1987, Die Anvendung von Spline-Funktionen auf die Positionsbestimung von Filamenten, Diplomarbeit, Institut für Astronomie der Universität Graz, 62 + suplement A (16 p.), B (23 p.)

Van Tend, W., \& Zwaan, C. 1976, in Basic Mechanism of Solar Activity, ed. V. Bumba, \& J. Kleczek, Proc. IAU Symp., 71, 45

Vršnak, B., Roša, D., Božič, H., et al. 1999, Sol. Phys., 187, 207 